\title{
Importance-Practice Analysis of University Professors' Teaching Competencies
}

\section{대학 교수의 교수역량에 대한 중요도-실행도(IPA) 분석}

\author{
Meera Park ${ }^{1}$, Nam Joo Je ${ }^{2}$, Jiwon Yoon ${ }^{3}$ \\ 박미라1, 제남주2, 윤지원 ${ }^{3}$ \\ ${ }^{1}$ Assistant Professor, Department of nursing, Changshin University, Korea, minerva32@cs.ac.kr \\ ${ }^{2}$ Associate Professor, Department of nursing, Changshin University, Korea, jnj4757@cs.ac.kr \\ ${ }^{3}$ Assistant Professor, Department of Korean Language Education, Changshin University, Korea, \\ jwyoon@cs.ac.kr
}

Corresponding author: Jiiwon Yoon

\begin{abstract}
This study analyzes the importance and practice perception of university professors' teaching competency which is intended to find ways to improve the quality of education and strengthen teaching competency. Data collection was conducted from June 30, 2021 to July 27, 2021 with 84 university professors nationwide using Google questionnaires. As for the research tool, Lee's 32-question teaching competency diagnosis tool was used. The characteristics of the subjects were analyzed by descriptive statistics. The gap between the importance and performance of the teaching competency recognized by the subject was analyzed as a paired t-test. The IPA (importance-practice analysis) Matrix analysis was performed to analyze the results of the difference between each variable. The areas that need to be maintained and strengthened were the 'basic comptence'. The 'teaching competence' appeared to be an area where further efforts were not needed and needs to be improved in the future. In addition, in the case of lecture management, teaching-learning design, and interaction, the importance was high, but the implementation was low, so it seemed that improvement efforts needed to be concentrated. Reflecting these results, it is necessary to establish an educational strategy faithful to basic competencies when preparing educational improvement measures to strengthen teaching competency. It is necessary to establish a strategy to modify the area of intensive improvement and a strategy to strengthen the area of maintenance and reinforcement.
\end{abstract}

Keywords: College Education, IPA, Teaching Competence, Strengthen Competence

요약: 본 연구는 대학교수가 인식하는 교수 역량에 대한 중요도와 실행도를 분석하여 교수역량 강화를 위한 교육 개선 방안을 모색하고자 시행하였다. 자료수집은 2021년 6월 30 일부터 7월 27일까지 구글 설문지를 사용하여 전국 84 명의 대학교수를 대상으로 실시되었다. 연구 도구로는 Lee의 32문항 교수 역량 진단 도구가 사용되었다. 대상자의 특성은 기술통계로 분석되었다. 대상자가 인식하는 교수 역량의 중요도와 실행도의 차이는 paried t-test로 분석하였다. IPA matrix 분석을 수행하여 서로 간의 차이에 대한 결과를 분석하였다. 연구 결과, 유지·강화되어야 할 부분은 '기본 역량'이었다. '교원 역량'은 더 이상의 노력이 필요하지 않고 앞으로 개선될 필요가 있는 분야로 보였다. 또한, 강의운영과

Received: August 16, 2021; 1st Review Result: October 01, 2021; 2nd Review Result: November 18, 2021 Accepted: December 31, 2021 
교수-학습 설계, 상호작용의 경우는 중요도는 높지만 실행도가 낮아 개선 노력이 집중될 필요가 있는 것으로 보였다. 이러한 결과를 반영하여, 교원 역량 강화를 위한 교육 개선 방안을 마련할 때 기본 역량에 충실한 교육 전략을 수립할 필요가 있다. 또한 중점 개선 분야 수정 전략과 정비·보강 분야 강화 전략 수립이 필요하다.

핵심어: 교수역량, 대학생, 역량강화, 중요도-실행도

\section{1. 서론}

지식기반 사회에서의 국가 경쟁력 확보를 위한 핵심적인 요소는 지식과 창의적인 인적 자원의 확보이다. 이러한 요구에 따라 세계 각국의 대학들은 그동안의 양적 팽창 위주의 대학발전에 대한 반성, 대학교육에 대한 비판적 검토를 통해 대학 교육의 경쟁력 강화와 질 향상을 위해 노력하고 있다. 이러한 노력은 다양한 측면에서 시행될 수 있겠으나 가장 먼저 고려되어야 될 것은 교육을 하는 교원의 역량이다[1]. 대학이 가지는 핵심 기능이자 본질은 가르치는 것, 즉 교수라고 할 수 있으며[2] 교수의 전문성 발전에 대한 연구는 이미 국내외에서 주목받는 이슈가 되었다[3]. 사회가 요구하는 학생을 키워내기 위한 교육이 효과적으로 이루어지기 위해서는 교수의 역할이 무엇보다 중요하기 때문이다.

특히 대학에서는 교수가 직접 교재를 선정하고 이를 바탕으로 수업을 설계하는 등 수업이 자율적으로 구성 및 운영되는 경향이 있으므로[4] 수업 실행의 주체인 교수는 수업의 질을 결정짓는 가장 중요한 요소이다. 그만큼 대학 교육의 질 제고에 있어서 교수의 역할은 매우 크다고 할 수 있다. 그러므로 대학교육의 질을 변화하고 확보하기 위해서는 대학교수들의 교수 역량을 강화해야 한다. 앞선 연구들도 우리나라 대학교육의 질적 개혁은 교수 역량의 증진으로부터 시작되어야 한다고 주장하고 있다[5].

현재 대학에서 교수역량의 증진을 위해 강의 평가제도와 교수학습센터의 설립이다. 그러나 교수역량을 평가하는 제도와 교수역량 및 향상을 도모하는 교수학습센터가 상호 연계하면서 시행되어야 효과가 극대화될 수 있을 것이다[6].

교수 역량은 성공적인 교육에 요구되는 지식·기술·태도 등의 총체로서 교수학습의 질에 영향을 미치는 주요 요인이다[7]. 교수역량은 크게 기본역량과 강의역량으로 나누어 생각할 수 있는데, 기본역량은 교수로서의 기본적인 책무와 관련하여 대학교육의 이해와 교육철학, 교수자의 보람, 윤리 의식 등에 관한 역량이며, 강의역량은 수업하는 교실 환경 뿐만 아니라 수업에 필요한 교육내용과 전략 설계, 학생 관리, 평가와 환류 등에 대한 역량을 의미한다. 아직 한국의 대학에서 교수학습의 질 관리는 교수의 교수역량 자체보다는 강의 평가 점수를 활용하는 것이 보편적이며, 강의 평가에 대한 신뢰도와 수용도가 낮은 편이어서 실제로 강의 평가 결과가 강의 개선에 반영되기 어렵다. 강의 평가를 해야만 학점 확인을 할 수 있는 강제적인 평가시스템으로 인해 학생들이 무성의한 답변을 하는 경우가 많아, 그 결과를 그대로 수용해야 하는지에 대해서 생각해보아야 한다[8-11]. 그리고 강의 평가는 학생들이 교수에게 주는 점수로 교수자 개인의 평가라기보다 수업 전반의 평가를 의미하기 때문에 교수 역량을 실질적으로 측정하는 데에는 한계가 따른다.

교수 역량 증진을 위해서는 무엇보다 교수 역량에 대한 정확한 진단이 선행되어야 
한다. 교수 역량 중 어떤 교수 역량이 필요하며 그것을 얼마나 하고 있는지, 또한 역량을 함양할 수 있게끔 하는 방안은 무엇일지 등에 대한 연구 또한 시급하다. 그동안 국내에서 이루어진 대학교수 역량 관련 연구는 대학 교원의 일반적인 교수 역량 분석 연구와 특정 전공이나 온라인교육 등 특수한 상황에서의 교수역량을 분석한 연구가 있었다. 또한 대학교원을 대상으로 교수 역량과 관련하여 실제 중요도-실행도를 활용한 요구분석 후 교수역량 강화를 위한 프로그램 개발 및 운영에 활용하기도 하였다[5][12].

그러나 해당 연구들은 대부분 2000년대 초반부터 2010년대에 집중적으로 이루어진 연구들로 현시점에서의 교수 역량에 대한 인식 확인은 지속해서 수행될 필요가 있으며 대학교수들의 역량에 대한 주기적인 진단은 교수 역량 진단 및 또한 일개 대학에 국한적으로 이루어졌던 연구 뿐만 아니라 대학 교원의 교수역량에 대한 면밀한 분석이 필요하다. 이에 본 연구는 IPA matrix 분석법을 활용하여 대학 교수가 지각하는 중요도실행도를 확인하고, 교수의 역량 강화를 위한 기초자료 마련에 도움이 되고자 한다.

\section{2. 연구방법}

\section{1 연구설계}

본 연구는 국내 대학에서 근무하는 교수의 역량에 대한 중요도와 실행도를 파악하기 위한 서술적 조사 연구이다.

\section{2 연구대상}

광고문으로 대상자를 모집하였으며, 국내 대학의 전임교수가 선정기준이고, 제외기준은 시간강사, 겸임교수, 초빙교수였다. 대상자 수는 $\mathrm{G}^{*}$ power 3.1.9 프로그램을 통해 효과 크기(effect size) 0.5(중간), 유의수준( a ) .05로 하였고, 검정력(1- $\beta$ ) 0.9, 양측 검정하였을 때 88 명이 산출되었으나, 96 명에게 자료 수집하여 응답이 불성실한 12 부를 제외한 84 부를 자료 분석에 이용하였다.

\section{3 연구도구}

본 연구의 교수역량은 Lee[2]가 개발한 교수역량 진단 도구를 사용하였다. 교수역량 진 단 도구 개발과 관련하여 이루어진 선행연구를 검토해 보았을 때, 대체로 많은 수의 문 항으로 구성되어 있는 반면,Lee[2]의 진단 도구는 타당도와 신뢰도를 확보하고,32개의 문 항으로 구성되어 실용성 역시 확보하여 교수역량을 진단하기에 적합하다고 판단하였다. 진단도구는 기본역량 8 문항, 강의역량 24 문항, 총 32 개의 문항으로 구성되어 있으며, 각 문항이 교수 활동의 효과성을 평가하는 데 얼마나 필요한 문항인지에 대한 중요도와 실 행도를 5점 Likert 척도로 응답할 수 있도록 자가진단 형식으로 구성되었다. 각 문항은 ‘전혀 그렇지 않다(1점)' 에서 '매우 그렇다(5점)' 의 5점 Likert 척도로 측정된다. 평균 점수가 높을수록 중요도와 실행도가 높음을 의미한다. 도구개발 당시 신뢰도 Cronbach's $a=.95$ 이었다. 본 연구에서 신뢰도 Cronbach's $a=.99$ 이었으며, 기본역량의 신뢰도 Cronbach's $a=.95$, 강의역량의 신뢰도 Cronbach's $a=.98$ 이었다. 


\section{4 윤리적 고려}

연구자는 먼저 연구목적과 절차, 대상자의 자율성과 권익 보호를 설명하고, 연구 참여 희망자에게 동의서를 받았다. 설문 소요 시간은 $10 \sim 15$ 분 정도이며 설문에 대한 응답은 익명 처리되고 언제든지 도중에 연구 참여를 자유의사에 따라 포기할 수 있고, 중도포기 시 불이익이 없다는 것을 설명하였다. 또한, 비밀과 익명이 보장됨을 설명하고 온라인 자 료는 password가 있는 파일에 저장하여 연구자 외의 접근을 제한하고 설문지 파일은 연 구종료 후 3 년간 보관한 후 삭제할 것이며 대상자의 개인정보와 자료는 숫자화 하여 연 구목적으로만 사용함을 설명하였다.

\section{5 자료 수집 기간 및 방법}

자료 수집은 2021년 6월 30일부터 2021년 7월 27일까지이며, 익명성과 편의성을 위해 구글(google) 온라인 설문으로 진행하였다. 먼저 SNS와 메일 등을 통해 대상자를 모집하 고, 연구 참여에 동의한 대상자에게 연구 참여 동의서와 온라인 설문 링크를 발송하였다.

\section{6 자료 분석}

자료 분석은 IBM/SPSS 25 통계프로그램을 이용하였고, 결과에 대한 유의수준은 .05로 측정하였다. 측정도구의 신뢰도를 실시하였다. 대상자의 특성은 서술적 통계(빈도 분석, 평균, 표준편차)로 구하였다. 대상자가 지각하는 교수역량의 중요도와 실행도의 상호차이 (Gap)는 paired t-test를 시행하였고, 교수역량의 중요도와 실행도의 상호차이 결과분석은 IPA Matrix로 분석하였다.

IPA(Importance Performance Analysis)는 행위의 중요도와 실행도를 스스로 평가하게 함으 로써 각 항목의 상대적인 중요도와 실행도를 동시에 비교 분석하는 평가기법으로, 평가 요소의 중요도와 실행도를 측정하여 그림 1 .과 같이 차원 도면상에 표시하고 그 위치에 따라 의미를 부여하는 분석법이다[13].

$\mathrm{A}$ 영역(지속적인 유지)은 중요도가 높고 실행도 역시 높은 것으로 이 영역에 속한 행위 는 계속 유지하는 것이 바람직하다. B영역(과잉노력지양)은 중요도가 낮은 것에 비해 실 행도가 높은 것을 의미하며, 실행을 줄이거나 투입될 노력을 다른 속성에 투입하도록 권 장한다. C영역(낮은 우선순위)은 중요도와 실행도 모두 낮은 것을 의미하며, 현재 이상의 노력이 불필요함을 의미한다. $\mathrm{D}$ 영역(중점개선: 노력 집중화의 지향)은 중요도는 높으나 실 행도가 낮은 것이며 향후 집중적으로 개선해야 하는 속성을 의미한다.

\section{7 연구의 제한점}

본 연구는 COVID-19로 인한 특수한 사회적 상황에서 진행되었으므로 신중한 해석과 주의가 필요하며, 전체 대학의 교수를 대상으로 하였으나 전체 대학 교수 수에 비해 응답률이 낮아 연구 결과를 확대하여 일반화함에 제한점이 있다. 


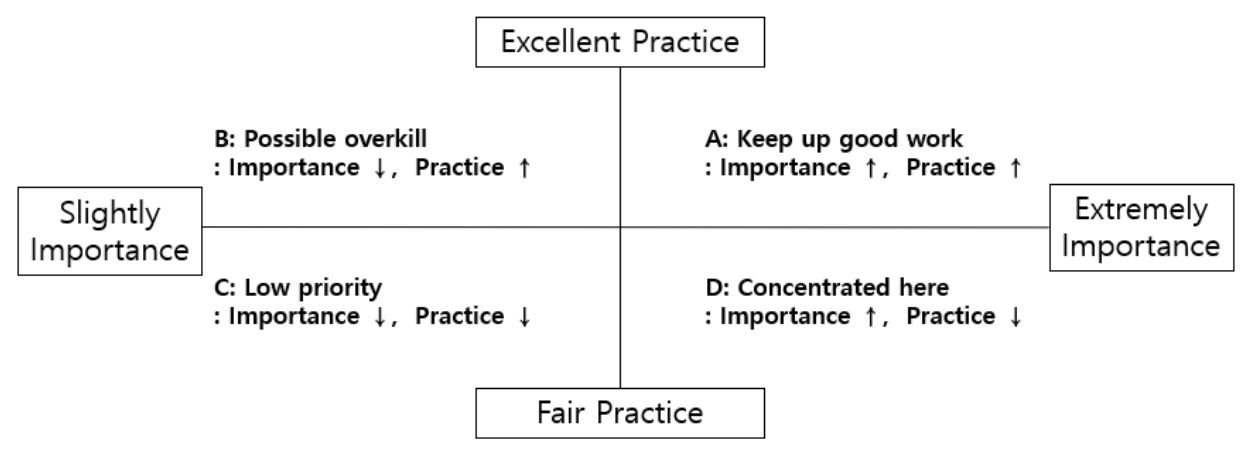

[그림 1] IPA 매트릭스

[Fig. 1] IPA Matrix

\section{3. 연구결과}

\section{1 대상자의 일반적 특성}

대상자는 남자 27 명 $(32.1 \%)$, 여자 57 명 $(67.9 \%)$ 이었다. 대상자의 평균연령은 46.93 세로 40 대(42.9\%)가 가장 많았고, 그 다음으로 50 대(29.8\%), 30대(20.2\%), 60대 이상(7.1\%) 순이었다. 경력은 평균 7.98 년이었으며, 5년 이하가 가장 많았고(46.4\%), 그 다음으로 6 10년(29.8\%), 16 20년(9.5\%) 순이었다. 직급의 경우 조교수(71.4\%)가 가장 많았으며, 전공계열의 경우 인문사회계열 $(50.0 \%)$ 이 가장 많았고, 그 다음으로 의약학계열 $(23.8 \%)$, 이학/공학계열(21.4\%). 예체능계열(4.8\%)순이었다[표 1].

\section{[표 1] 대상자의 일반적 특성}

[Table 1] Characteristics of Participants ( $\mathrm{N}=84)$

\begin{tabular}{|c|c|c|}
\hline Characteristics & Categories & $\mathrm{n}(\%)$ \\
\hline \multirow{2}{*}{ Gender } & Male & $27(32.1)$ \\
\hline & Female & $57(67.9)$ \\
\hline \multirow{4}{*}{$\begin{array}{c}\text { Age }(y r) \\
\text { Mean } \pm \text { SD: } 46.93 \pm 7.59\end{array}$} & $30-39$ & $17(20.2)$ \\
\hline & $40-49$ & $36(42.9)$ \\
\hline & $50-59$ & $25(29.8)$ \\
\hline & $\geq 60$ & $6(7.1)$ \\
\hline \multirow{5}{*}{$\begin{array}{c}\text { Career(yr) } \\
\text { Mean } \pm \text { SD: } 7.98 \pm 6.61\end{array}$} & $\leq 5$ & $39(46.4)$ \\
\hline & $6-10$ & $25(29.8)$ \\
\hline & $11-15$ & $7(8.3)$ \\
\hline & $16-20$ & $8(9.5)$ \\
\hline & $\geq 21$ & $5(6.0)$ \\
\hline School position & Assistant Professor & $60(71.4)$ \\
\hline
\end{tabular}




\begin{tabular}{|l|c|c|}
\hline \multirow{4}{*}{} & Associate Professor & $13(15.5)$ \\
\cline { 2 - 3 } & Professor & $11(13.1)$ \\
\hline \multirow{4}{*}{ Major } & Humanities society & $42(50.0)$ \\
\cline { 2 - 3 } & Science/Engineering & $18(21.4)$ \\
\cline { 2 - 3 } & Medicine & $20(23.8)$ \\
\cline { 2 - 3 } & Art and Physical & $4(4.8)$ \\
\hline
\end{tabular}

\section{2 교수 역량의 중요도와 실행도의 차이}

대상자의 교수역량의 중요도와 실행도의 상호차이는 [표 2]와 같다. 교수역량은 크게 기본역량과 강의역량으로 나누어진다. 대상자가 인지하는 교수역량의 중요도는 평균 4.30점(5점 만점)이었으며, 영역으로 구분하여 살펴보았을 때, 기본역량은 평균 4.39점(5점 만점), 강의역량은 평균 4.21점(5점 만점)으로 강의역량보다 기본역량이 더 중요하다고 응답하였다.

교수역량의 실행도는 평균 3.84점(5점 만점)이었으며, 영역 중 기본역량의 경우 평균 4.06점(5점 만점), 강의역량의 경우 평균 3.63점(5점 만점)으로 강의역량보다 기본역량을 더 실행하고 있다고 하였다.

중요도가 가장 높은 항목은 '나는 대학 교수로서 신분에 합당한 윤리규범을 준수한다였으며 중요도가 가장 낮은 항목은‘나는 학습목표를 달성하기 위한 평가 계획을 세우고 적절한 평가도구를 개발한다'였다. 한편 실행도에 대해서는 '나는 대학 교수로서 신분에 합당한 윤리규범을 준수한다'에 대한 점수가 가장 높았고, '나는 학생들에게 과제물에 대한 피드백을 곧바로 해 준다’에 대한 점수가 가장 낮은 것으로 나타났다.

중요도와 실행도 간의 차이가 가장 큰 항목은 '과제물 피드백, 과제물 점검, 수업을 위한 교재 연구 및 내용 분석, 순이었다.

전체 중요도는 실행도보다 높아 통계적으로 유의한 차이가 있었으며, 기본역량과 강의역량 모두 중요도와 실행도의 차이가 있었다 $(\mathrm{p}<.05)$. 세부 속성별로 살펴보았을 때, 24 개 문항 모두에서 중요도와 실행도 간의 통계적으로 유의한 차이가 있었다( $\mathrm{p}<.05)$.

\section{[표 2] 교수 역량의 중요도와 실행도 차이}

[Table 2] The Difference between the Importance and Practice of Teaching Competency $\quad(\mathrm{N}=84)$

\begin{tabular}{|c|c|c|c|c|c|c|}
\hline & \multirow{2}{*}{ Competency indicator } & Importance & Practice & Gap & \multirow{2}{*}{$\mathrm{t}$} & \multirow{2}{*}{$p$} \\
\hline & & Mean \pm SD & Mean \pm SD & Mean \pm SD & & \\
\hline \multirow{5}{*}{$\begin{array}{l}\text { Basic } \\
\text { competency }\end{array}$} & $\begin{array}{l}\text { 1. I have a clear view of teaching as a } \\
\text { professor. }\end{array}$ & $4.49 \pm 0.86$ & $4.06 \pm 0.78$ & $0.43 \pm 0.07$ & 6.47 & $<.001$ \\
\hline & $\begin{array}{l}\text { 2. I have sufficient knowledge and deep } \\
\text { understanding of the subject matter. }\end{array}$ & $4.40 \pm 0.87$ & $4.14 \pm 0.76$ & $0.26 \pm 0.07$ & 3.98 & $<.001$ \\
\hline & $\begin{array}{l}\text { 3. I continuously research and utilize } \\
\text { knowledge related to the subject. }\end{array}$ & $4.49 \pm 0.83$ & $4.08 \pm 0.93$ & $0.41 \pm 0.08$ & 4.97 & $<.001$ \\
\hline & $\begin{array}{l}\text { 4. I abide by the code of ethics appropriate } \\
\text { to my status as a university professor. }\end{array}$ & $4.55 \pm 0.84$ & $4.41 \pm 0.84$ & $0.13 \pm 0.04$ & 2.97 & .004 \\
\hline & $\begin{array}{l}\text { 5. I actively participate in pedagogical } \\
\text { improvement programs that help improve } \\
\text { teaching. }\end{array}$ & $4.10 \pm 0.90$ & $3.69 \pm 0.97$ & $0.41 \pm 0.08$ & 5.33 & $<.001$ \\
\hline
\end{tabular}




\begin{tabular}{|c|c|c|c|c|c|c|}
\hline & $\begin{array}{l}\text { 6. I explore and try new teaching methods } \\
\text { for better teaching. }\end{array}$ & $4.17 \pm 0.80$ & $3.63 \pm 0.99$ & $0.54 \pm 0.08$ & 6.40 & $<.001$ \\
\hline & $\begin{array}{l}\text { 7. I try to recognize and respect the diversity } \\
\text { of my students. }\end{array}$ & $4.49 \pm 0.80$ & $4.33 \pm 0.86$ & $0.16 \pm 0.06$ & 2.59 & .011 \\
\hline & 8. I often talk to my students. & $4.43 \pm 0.87$ & $4.11 \pm 0.97$ & $0.32 \pm 006$ & 5.44 & $<.001$ \\
\hline & Sub-tatal & $4.39 \pm 0.74$ & $4.06 \pm 0.68$ & $0.33 \pm 0.04$ & 8.16 & $<.001$ \\
\hline \multirow{22}{*}{$\begin{array}{l}\text { Teaching } \\
\text { Copetency }\end{array}$} & $\begin{array}{l}\text { 9. I thoroughly prepare class materials and } \\
\text { media necessary for class. }\end{array}$ & $4.37 \pm 0.82$ & $3.82 \pm 0.89$ & $0.55 \pm 0.09$ & 6.29 & $<.001$ \\
\hline & $\begin{array}{l}\text { 10. I plan in advance the goals I want to } \\
\text { achieve in class. }\end{array}$ & $4.20 \pm 0.79$ & $3.83 \pm 0.86$ & $0.37 \pm 0.06$ & 6.10 & $<.001$ \\
\hline & $\begin{array}{l}\text { 11. I thoroughly research the textbook and } \\
\text { analyze the content for the class. }\end{array}$ & $4.35 \pm 0.87$ & $3.75 \pm 0.88$ & $0.60 \pm 0.08$ & 7.47 & $<.001$ \\
\hline & $\begin{array}{l}\text { 12. I organize the lecture content } \\
\text { systematically so that the content can be } \\
\text { easily delivered.. }\end{array}$ & $4.25 \pm 0.83$ & $3.93 \pm 0.86$ & $0.32 \pm 0.07$ & 4.58 & $<.001$ \\
\hline & $\begin{array}{l}\text { 13. I plan teaching methods and strategies } \\
\text { considering the level and characteristics of } \\
\text { students. }\end{array}$ & $4.30 \pm 0.86$ & $3.87 \pm 0.91$ & $0.44 \pm 0.09$ & 5.17 & $<.001$ \\
\hline & $\begin{array}{l}\text { 14. I decide the scope and difficulty of } \\
\text { lecture content by reflecting the students' } \\
\text { pre-learning level or requirements. }\end{array}$ & $4.18 \pm 0.89$ & $3.79 \pm 0.95$ & $0.39 \pm 0.08$ & 5.06 & $<.001$ \\
\hline & $\begin{array}{l}\text { 15. I check the students' pre-learning status } \\
\text { at the start of class. }\end{array}$ & $4.05 \pm 0.92$ & $3.63 \pm 0.97$ & $0.42 \pm 0.08$ & 5.22 & $<.001$ \\
\hline & $\begin{array}{l}\text { 16. I explain why I present the assignments } \\
\text { to my students.. }\end{array}$ & $4.15 \pm 0.75$ & $3.63 \pm 1.08$ & $0.52 \pm 0.11$ & 4.92 & $<.001$ \\
\hline & 17. I thoroughly check students' work. & $4.26 \pm 0.82$ & $3.39 \pm 1.26$ & $0.87 \pm 0.15$ & 5.82 & $<.001$ \\
\hline & $\begin{array}{l}\text { 18. I present students with a connection } \\
\text { between what they have already learned and } \\
\text { what they will learn. }\end{array}$ & $4.13 \pm 0.80$ & $3.85 \pm 0.86$ & $0.29 \pm 0.07$ & 4.42 & $<.001$ \\
\hline & $\begin{array}{l}\text { 19. I present the overall outline of the class } \\
\text { concisely at the beginning of the class. }\end{array}$ & $4.18 \pm 0.81$ & $3.86 \pm 0.98$ & $0.32 \pm 0.08$ & 4.13 & $<.001$ \\
\hline & $\begin{array}{l}\text { 20. I summarize or outline class content } \\
\text { before class ends. }\end{array}$ & $3.98 \pm 0.91$ & $3.68 \pm 1.04$ & $0.30 \pm 0.09$ & 3.46 & $<.001$ \\
\hline & $\begin{array}{l}\text { 21. I provide students with a wealth of } \\
\text { examples/cases/information for effective } \\
\text { instruction. }\end{array}$ & $4.26 \pm 0.91$ & $3.97 \pm 0.94$ & $0.32 \pm 0.06$ & 5.04 & $<.001$ \\
\hline & $\begin{array}{l}22 . \text { I use a variety of media and materials to } \\
\text { make class fun or help students understand. }\end{array}$ & $4.20 \pm 0.90$ & $3.86 \pm 0.84$ & $0.35 \pm 0.08$ & 4.21 & $<.001$ \\
\hline & $\begin{array}{l}\text { 23. I create an atmosphere in which students } \\
\text { naturally express their thoughts and ask } \\
\text { questions. }\end{array}$ & $4.19 \pm 0.87$ & $3.76 \pm 0.94$ & $0.43 \pm 0.08$ & 5.36 & $<.001$ \\
\hline & $\begin{array}{l}\text { 24. I often ask questions that arouse } \\
\text { students' intellectual curiosity. }\end{array}$ & $4.06 \pm 0.86$ & $3.65 \pm 0.91$ & $0.41 \pm 0.09$ & 4.43 & $<.001$ \\
\hline & $\begin{array}{l}\text { 25. I give students direct feedback on } \\
\text { assignments. }\end{array}$ & $3.99 \pm 0.93$ & $3.08 \pm 1.21$ & $0.91 \pm 0.14$ & 6.31 & $<.001$ \\
\hline & $\begin{array}{l}\text { 26. I provide appropriate answers and } \\
\text { feedback to students' questions. }\end{array}$ & $4.20 \pm 0.86$ & $3.86 \pm 0.97$ & $0.35 \pm 0.10$ & 3.63 & $<.001$ \\
\hline & $\begin{array}{l}27 . \text { I encourage students to be confident in } \\
\text { class. }\end{array}$ & $4.32 \pm 0.88$ & $4.05 \pm 0.97$ & $0.27 \pm 0.07$ & 3.77 & $<.001$ \\
\hline & $\begin{array}{l}\text { 28. I motivate students to have a long-term } \\
\text { vision. }\end{array}$ & $4.38 \pm 0.86$ & $4.18 \pm 0.93$ & $0.20 \pm 0.07$ & 2.76 & .007 \\
\hline & $\begin{array}{l}\text { 29. I motivate students to study on their } \\
\text { own. }\end{array}$ & $4.32 \pm 0.92$ & $3.94 \pm 0.87$ & $0.38 \pm 0.07$ & 5.17 & $<.001$ \\
\hline & $\begin{array}{l}\text { 30. I develop an assessment plan to achieve } \\
\text { learning goals and develop appropriate } \\
\text { assessment tools. }\end{array}$ & $3.86 \pm 0.92$ & $3.43 \pm 0.87$ & $0.43 \pm 0.09$ & 4.76 & $<.001$ \\
\hline
\end{tabular}




\begin{tabular}{|l|l|c|c|c|c|c|}
\hline & $\begin{array}{l}\text { 31. I clearly inform students of the standards } \\
\text { and methods of evaluation and evaluate } \\
\text { them fairly. }\end{array}$ & $4.45 \pm 0.86$ & $4.18 \pm 0.93$ & $0.27 \pm 0.07$ & 4.00 & $<.001$ \\
\cline { 2 - 7 } & $\begin{array}{l}\text { 32. After class, I constantly reflect on what I } \\
\text { did well and what I need to improve. }\end{array}$ & $4.33 \pm 0.88$ & $3.93 \pm 0.90$ & $0.41 \pm 0.09$ & 4.68 & $<.001$ \\
\cline { 2 - 7 } & Sub-tatal & $4.21 \pm 0.72$ & $3.63 \pm 0.63$ & $0.58 \pm 0.05$ & 11.17 & $<.001$ \\
\hline Total & $4.30 \pm 0.72$ & $3.84 \pm 0.63$ & $0.45 \pm 0.38$ & 11.00 & $<.001$ \\
\hline
\end{tabular}

\subsection{IPA 분석}

교수역량의 중요도와 실행도를 분석한 결과는 [그림 2], [그림 3]과 같다.

분석 결과, 계속 유지·강화해야 할 부분은 '기본역량' 이었으며, '강의역량' 은 더 이상의 노력 투입이 불필요한 것으로 나타났다.

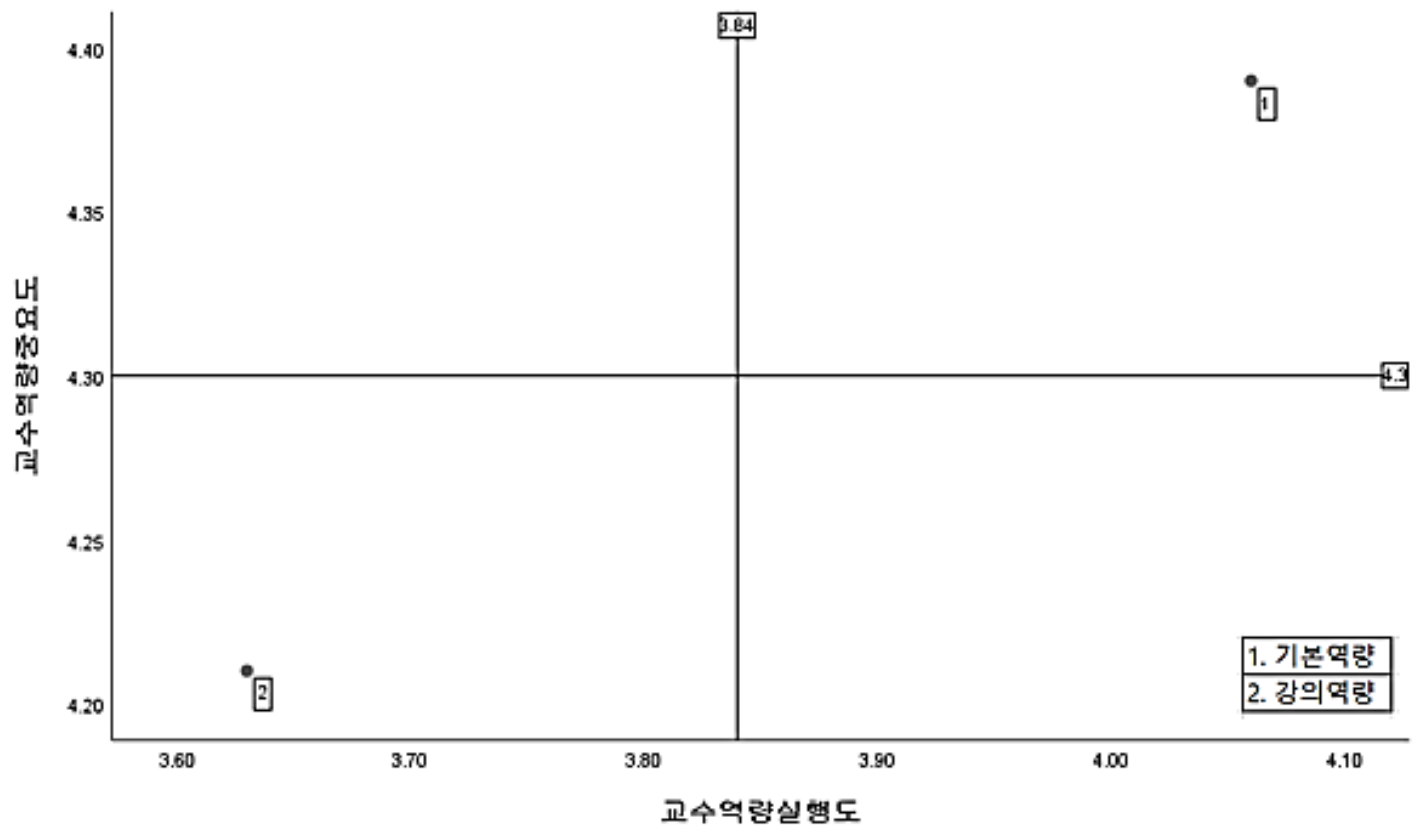

[그림 2] 중요도와 실행도의 영역에 따른 매트릭스

[Fig. 2] Matrix Based on Areas of Importance and Performance

세부 속성별 분석 결과는 다음과 같다. 우선, (1)나는 교수로서 가르치는 것에 대한 분명한 교육관을 가지고 있다, (2)나는 교과내용에 대한 충분한 지식과 깊은 이해를 가지고 있다, (3)나는 교과와 관련된 지식을 지속적으로 연구하고 활용한다, (4)나는 대학교수로서 신분에 합당한 윤리규범을 준수한다, (7)나는 학생들의 다양성을 인정하고 존중하려 노력한다, (8)나는 평소 학생들과 자주 대화를 한다, (13)나는 학생들의 수준 및 특성을 고려하여 교수방법 및 전략을 계획한다, (27)나는 수업에서 학생들이 자신감을 갖도록 격려한다, (28)나는 학생들이 장기적인 비전을 갖도록 동기부여를 한다, (29)나는 학생들이 스스로 공부할 수 있도록 동기부여를 한다, (31)나는 학생들에게 평가의 기준 및 방법을 명확히 알려주고 공정하게 평가한다, (32)나는 수업을 마친 후 잘된 점과 개선할 점에 대해 꾸준히 성찰하고 개선한다의 속성이 '계속 유지·강화해야 할 영역' 인 
1 '사분면'에 포함되었다.

‘2사분면' 인 ‘수행도를 줄이거나 투입될 노력을 다른 평가속성에 투입하도록 권장하는 영역에는 (9)나는 수업에 필요한 수업자료와 매체를 철저히 준비한다, (11)나는 수업을 위한 교재 연구 및 내용분석을 철저히 한다의 속성이 포함되었다.

'3사분면' 인 '더 이상의 노력투입이 불필요한 영역 '에는 (5)나는 강의 개선에 도움이 되는 교수법 향상 프로그램에 적극적으로 참여한다, (6)나는 더 나은 수업을 위해 새로운 교수법을 탐구하고 시도한다, (10)나는 수업에서 성취하고자 하는 목표를 사전에 구상한다, (14)나는 학생들의 사전학습 수준이나 요구사항을 반영하여 강의내용의 범위와 난이도를 결정한다, (15)나는 수업시작 시 학생들의 사전학습 상태를 확인한다, (16)나는 학생들에게 과제를 제시하는 이유에 대해 설명한다, (17)나는 학생들의 과제물을 철저히 점검한다, (20)나는 수업종료 전에 수업내용을 요약하거나 개괄한다, (23)나는 학생들이 자신의 생각을 자연스럽게 표현하고 질문하도록 분위기를 만든다, (24)나는 학생들의 지적인 호기심을 유발할 수 있는 질문을 자주 한다, $(25)$ 나는 학생들에게 과제물에 대한 피드백을 곧바로 해준다, (30)나는 학습목표를 달성하기 위한 평가 계획을 세우고 적절한 평가도구를 개발한다의 속성이 포함되었다.

‘4사분면' 인 ‘중요도에 비해 실행도가 낮은 영역에 속하여 더 많은 수행이 요구' 되는 영역에는 (12)나는 교과내용을 쉽게 전달할 수 있도록 강의내용을 체계적으로 구성한다, (18)나는 학생들이 이미 학습한 내용과 새롭게 배울 내용을 연결시켜 제시한다, (19)나는 수업시작 시 본 수업의 전반적인 윤곽을 간결하게 제시한다, (21)나는 효과적인 수업을 위해 풍부한 예시/사례/정보를 학생들에게 제공한다, (22)나는 수업을 재미있게 하거나 학생들의 이해를 돕기 위해 다양한 매체와 자료를 활용한다, (26)나는 학생들의 질문에 적절한 답변과 피드백을 제공한다의 속성이 포함되었다.

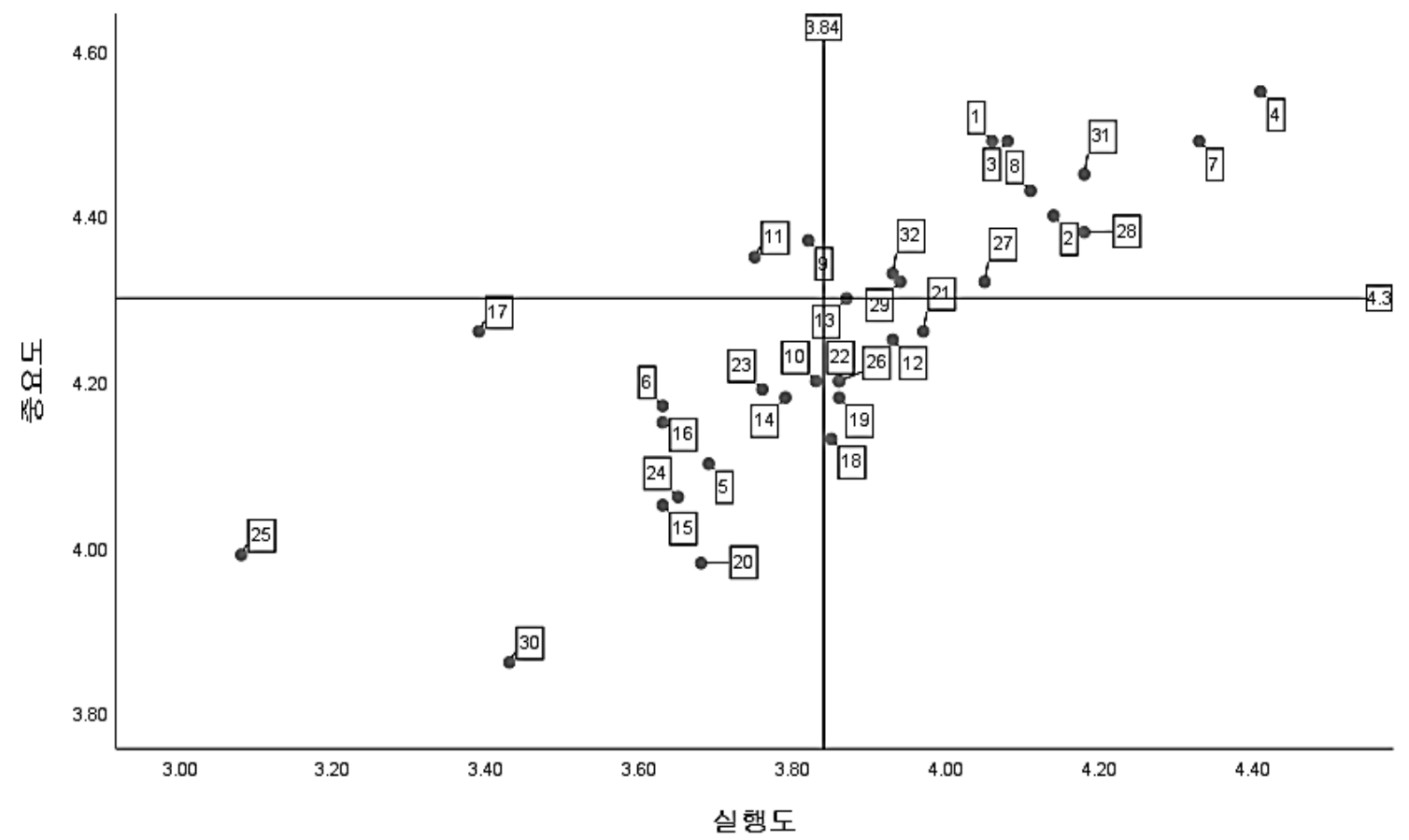

[그림 3] 중요도와 실행도의 세부영역에 따른 매트릭스

[Fig. 3] Matrix based on the Detailed Areas of Importance and Performance 


\section{4. 결론 및 제언}

본 연구는 국내 대학 교수들의 교수 역량에 대한 중요도와 실행도의 차이를 분석하고, 개선해야 할 방안을 모색하고자 시도되였다. ‘중요도와 실행도 차이 분석'과 ‘중요도와 실행도 분석'의 두 가지 측면에서 연구의 결과를 살펴보면 다음과 같다.

먼저, ‘중요도와 실행도 차이 분석'의 경우, 기본역량과 강의역량에 대해서 중요도의 평균이 실행도보다 높았으며, 중요도와 실행도의 차이는 모든 영역에서 통계적으로 유의하였다. 이 결과는 전반적으로 교육의 실행 수준을 높일 수 있는 방안을 마련해야 한다는 사실을 입증해 줌과 동시에 각 영역별로 특별히 고려해야 할 사항을 파악하여 대처하는 노력이 투입되어야 할 필요성을 시사한다.

'나는 대학 교수로서 신분에 합당한 윤리 규범을 준수한다' 가 중요도와 실행도 모두 가장 높았다는 점에서 교수들이 교수로서의 윤리 규범 준수를 중시하고 있고, 그 인식이 실행까지 이어졌음을 알 수 있다. '나는 학습목표를 달성하기 위한 평가 계획을 세우고 적절한 평가도구를 개발한다' 에 대한 중요도는 가장 낮았는데, 그 다음 문항인 '나는 학생들에게 평가의 기준 및 방법을 명확히 알려주고 공정하게 평가한다' 의 점수는 높았다. 평가 도구 개발에 대하여 시험 문제 출제 등은 교수의 당연한 역할로 평가 계획 및 도구 개발에 대해 상대적으로 중요하게 여기지 않고 있음을 알 수 있다. 또한, '나는 학생들에게 과제물에 대한 피드백을 곧바로 해 준다' 에 대한 점수가 가장 낮은 것으로 나타났는데, 실제로 과제물 제시뿐만 아니라 과제물에 대한 피드백의 중요성을 알고 개선되어야 할 것이다.

또한, IPA 분석에 따른 중요도-실행도 매트릭스를 살펴본 결과, 기본역량은 중요도와 수행도가 모두 높아 계속 유지 - 강화해야 하지만, 강의역량은 중요도와 실행도가 모두 낮아 더 이상의 노력 투입이 불필요한 영역으로 나타났다.

4 가지 영역 중에서 가장 생각해야 할 부분은 개선 노력 집중영역의 요소들이다. 이 요소들에 대해 교수들은 중요도는 높게 인식하지만 실행도가 낮으므로 개선 노력을 집중해야 한다. 중요도는 높으나 실행도가 낮은 4사분면에 속한 항목은 대체로 강의운영 $(18,19,21,22)$ 과 관련된 항목이며 교수-학습 설계(12)와 상호작용(26)에 포함되는 것이었다.

중요도-실행도 분석을 통해 확인된 개선 노력 집중 요소에 우선순위를 두고 중요도와 실행도가 모두 높은 항목은 그 상태를 유지하며, 중요도에 비해 수행도가 높은 항목은 지나치게 많은 노력을 기울이지 않도록 하는 방향으로 교수 역량 강화 교육이 개선되어야 할 것이다. 본 연구 결과를 교수 역량 강화 교육에 반영함에 있어 다방면으로 노력을 기울여야 할 것이다.

본 연구에서 확인한 내용은 COVID-19라는 특수한 시대적 상황에서 시행된 연구이므로 본 연구결과를 해석하고 일반화함에 제한이 있다. 본 연구 결과를 바탕으로 다음과 같은 제언을 하고자 한다. 첫째, 본 연구는 국내 대학의 교수를 대상으로 진행된 연구로서 희망자에 한해 연구를 진행하였으므로 일반화에 제한점이 있으므로 추가 연구를 제언한다. 둘째, 본 연구는 교수자 개인이 지각하는 중요도와 실행도에 기초하여 자가진단으로 이루어졌기에 한계점이 있으므로 해당 내용을 보완할 수 있는 추가 연구를 제언한다. 셋째, 본 연구 결과는 일반적인 교수 역량을 다루고 있기에 전공 및 계열에 따른 차별화가 반영되지 못하였으므로 이에 대한 추가 연구를 제언한다. 넷째, 대학 교수의 중요도와 실행도의 차이에 따른 결과를 보완할 수 있는 프로그램 개발과 효과를 
확인하는 추가 연구를 제언한다.

\section{References}

[1] M. M. Kowal, Evolution of a profession: A review of the community engagement professional in higher education: A competency model for an emerging field, The International Journal of Research on Service-Learning and Community Engagement, (2017), Vol.5, No.1, pp.181-183.

[2] Y. R. Lee, Development of a Diagnostic Tool for teaching Competency of Faculty, Keimyung University, Master's Thesis, pp.57-81, (2018)

[3] Y. Liu, Perceptions of university faculty on the importance and satisfaction of Faculty Development Center, Keimyung University, Doctoral dissertation, pp.63-70, (2020)

[4] K. W. Chang, The analysis of characteristics of good teaching using appreciative inquiry, Korean Education Inquiry, (2014), Vol.32, No.1, pp.137-160, UCI: G704-SER000014634.2014.32.1.008

[5] H. L. Roh, M. N. Choi, The development of the teaching competency model for HRD (Human Resources Development), Journal of Vocational Education \& Training, (2004), Vol.7, No.2, pp.1-28, UCI: G704-000976.2004.7.2.004

[6] E. H. Yang, J. Chung, Needs Analysis for Improving Teaching Competency, Journal of Educational Technology, (2010), Vol.26, No.2, pp.25-52, UCI: G704-000394.2010.26.2.002

[7] D. E. Tigelaar, D. H. Dolmans, I. H. Wolfhagen, C. P. Vander Vleuten, The development and validation of a framework for teaching competencies in higher education, Higher education, (2004), Vol.48, No.2, pp.253-268, DOI: https://doi.org/10.1023/B:HIGH.0000034318.74275.e4

[8] K. S. Yang, Meta-research on the influence of course, instructor, and student characteristics in student evaluation of teaching at universities, The Korean Journal of Educational Methodology Studies, (2014), Vol.26, No.2, pp.293-322, DOI: $10.17927 /$ tkjems.2014.26.2.293

[9] M. S. Cho, C. S. Kim, The relationship between the satisfaction levels of education and clinical practice in the dental hygiene students, Journal of Korean society of Dental Hygiene, (2014), Vol.14, No.5, pp.649-657, DOI: 10.13065/jksdh.2014.14.05.649

[10] S. Shin, J. Kwon, A study on the improvement of the reliability of the course evaluation : Focused on the management of course evaluation system, Soonchunhyang Journal of Humanities, (2016), Vol.35, No.4, pp.115-145, UCI: G704SER000013584.2016.35.4.003

[11] S. H. Ahn, S. J. Lee, Investigation on Methods to Improve the Reliability of Education Satisfaction Surveys: Focused on the S University Case, Journal of Digital Convergence, (2017), Vol.15, No.12, pp.71-77, DOI: 10.14400/JDC.2017.15.12.71

[12] M. R. Eom, A Suggestion on Teaching Support Program through the Competencies analysis of University Faculties' Technology, Pedagogy, and Content Knowledge(TPACK), Theory and Practice of Education, (2012), Vol.17, No.3, pp.21-45, UCI: G704-001913.2012.17.3.005

[13] J. A. Martilla, J. C. James, Importance-Performance Analysis, Journal of Marketing, (1977), Vol.41, No.1, pp.77-79, DOI: https://doi.org/10.2307/1250495 